\title{
Development and production of hard X-ray multilayer optics for HEFT
}

Jason E. Koglin, Finn Erland Christensen, Jim Chonko, William W. Craig, Todd R. Decker, et al.

Jason E. Koglin, Finn Erland Christensen, Jim Chonko, William W. Craig, Todd R. Decker, Mario A. Jimenez-Garate, Kurt S. Gunderson, Charles J. Hailey, Fiona A. Harrison, Carsten P. Jensen, Mike Sileo, David L. Windt, Haitao Yu, "Development and production of hard X-ray multilayer optics for HEFT," Proc. SPIE 4851, X-Ray and Gamma-Ray Telescopes and Instruments for Astronomy, (11 March 2003); doi: 10.1117/12.461479

Event: Astronomical Telescopes and Instrumentation, 2002, Waikoloa, Hawai'i, United States 


\title{
Development and production of hard X-ray multilayer optics for HEFT
}

\author{
Jason E. Koglin ${ }^{a}$, Finn E. Christensen ${ }^{c}$, Jim Chonko $^{a}$, William W. Craig ${ }^{d}$, \\ Todd R. Decker ${ }^{d}$, Mario A. Jimenez-Garate ${ }^{a *}$, Kurt Gunderson ${ }^{d}$, Charles J. Hailey ${ }^{a}$, \\ Fiona A. Harrison ${ }^{b}$, Carsten P. Jensen ${ }^{c}$, Mike Sileo ${ }^{a}$, David L. Windt ${ }^{a}$, Haitao $\mathrm{Yu}^{a}$ \\ ${ }^{a}$ Columbia Astrophysics Laboratory, New York, NY \\ ${ }^{b}$ California Institute of Technology, Pasadena, CA \\ ${ }^{c}$ Danish Space Research Institute, Copenhagen, Denmark \\ ${ }^{d}$ Lawrence Livermore National Laboratory, Livermore, CA
}

\begin{abstract}
The High Energy Focusing Telescope (HEFT) will observe a wide range of objects including young supernova remnants, active galactic nuclei, and galaxy clusters at energies between 20 and $70 \mathrm{keV}$. Large collecting areas are achieved by tightly nesting layers of grazing incidence mirrors in a conic approximation Wolter-I design. The segmented mirrors that form these layers are made of thermally formed glass substrates coated with depthgraded multilayer films for enhanced reflectivity. The mirrors are assembled using an over-constraint method that forces the overall shape of the nominally cylindrical substrates to the appropriate conic form. We will present performance data on the HEFT optics and report the current status of the assembly production.
\end{abstract}

Keywords: X-ray optics, Hard X-ray telescope

\section{SCIENCE GOALS AND OPTICS REQUIREMENTS}

The High Energy Focusing Telescope (HEFT) is a balloon-borne experiment employing focusing optics in the hard X-ray band (20 - $70 \mathrm{keV}$ ) for sensitive observations of astrophysical sources. In the past, coded aperture or collimated optics instruments were used in hard X-ray experiments. For these non-focusing instruments, the detector area is actually slightly greater than the effective collecting area. Focusing experiments, however, concentrate the incoming flux onto much smaller detectors, which reduces the total detector background. In addition to providing higher angular resolution, this greatly improves the detector signal to noise ratio making much fainter astronomical sources detectable. We have developed thermally formed glass and a unique mounting approach to build optic modules with large effective areas. Some of the primary scientific objectives for HEFT include imaging and spectroscopy of ${ }^{44} \mathrm{Ti}$ emission in young supernova remnants, sensitive observations of obscured Active Galactic Nuclei, spectroscopic observations of accreting high-magnetic field pulsars, and mapping the non-thermal emissia in the Galactic Center.

These science objectives motivate the optics design toward an approach that emphasizes building telescopes with large collecting areas that are capable of operating over a broad energy band. This is true not only for HEFT but also for future hard X-ray satellite missions under design (e.g., Constellation-X and XEUS). Relatively large collecting areas are achieved by current soft X-ray telescopes such as Chandra and XMM using nested Wolter-I mirror assemblies that focus X-rays through total external reflection. Expanding this approach to hard X-rays is challenging because the critical angle (the maximum incidence angle at which total external reflection occurs) is inversely proportional to the photon energy. Thus, very small incidence angles are required to reflect high-energy X-rays. However, multilayer coatings consisting of alternating layers of high and low density materials can be used to achieve high reflectivities at angles greater than the critical graze angle through constructive interference at multiple surface interfaces. Depth-graded multilayer coatings vary the thickness of these alternating layers to achieve high reflectivity over a broad range of energies.

Correspondence: koglin@astro.columbia.edu, http://astro.columbia.edu/ koglin

* Current address: Massachusetts Institute of Technology, Cambridge, MA 
Several approaches have been taken to build soft X-ray mirrors in the past. Aluminum foils (ASCA, ${ }^{1}$ ASTRO-E, ${ }^{2}$ SODART $^{3,4}$ ), electroformed nickel from replication $\left(\mathrm{XMM}^{-N e w t o n}{ }^{5}\right.$ ) and monolithic Zerodur $\left(\right.$ Chandra $\left.^{6}\right)$ have been successfully used as optic substrates. We have taken a different approach and have developed thermally-formed glass substrates and an overconstraint mounting technique. ${ }^{7}$ The mounting technique relies on using our segmented mirrors to form each mirror layer. However, our approach is flexible enough to accommodate almost any size of segmented substrate. Not only does our assembly approach meet the needs of HEFT, it also has the potential to meet the design criteria for future Explorer class satellites and major space based observatories. In this paper, we first summarize the HEFT optics design. Next, each step in the optics production process is described. Then, results of prototype development will be presented. Finally, the our progress in producing flight-grade optics modules for HEFT is given.

\section{HEFT DESIGN}

The overall design of the HEFT telescope calls for an array of co-aligned, conical-approximation Wolter I mirror assemblies. Each of these optics modules focuses hard X-rays $(20-70 \mathrm{keV})$ onto an individual, shielded, solid-state Cadmium Zinc Telluride (CdZnTe) pixel detectors. ${ }^{8} \mathrm{~W} / \mathrm{Si}$ depth-graded multilayer coatings provide high-energy reflectivity at reasonable graze angles, with response extending to $70 \mathrm{keV} .{ }^{9}$ Later telescopes will provide good energy response up to $100 \mathrm{keV}$ for the innermost shells using other multilayer coatings. The 0.3 $\mathrm{mm}$ thin shells form nested mirrors that are separated from the focal plane detectors by 6 meters. The HEFT design allows for up to fourteen modules to be mounted on a precision balloon pointing platform. The design calls for optics with an angular resolution of 1' half-power diameter (HPD) and a field of view (FOV) of 17'. The telescope geometry is optimized by balancing on-axis and off-axis collecting area ${ }^{10,11}$ In doing so, each layer will partially obscure the next layer for off-axis photons of certain incidence angles (i.e., vignetting).

In preparation for a 2003 flight, the instrument components are being prepared for full integration on the HEFT balloon gondola. Figure 1 shows the gondola in the center surrounded by more detailed pictures of the major HEFT components. The star tracker, control components and electronics are provided by LLNL. The CdZnTe detectors with their flight shields and the pressurized Kevlar dome in which they will be housed, along with the overall flight management, is provided by Caltech. The flight optics modules are being fabricated, coated and tested by Columbia University, DSRI, and Colorado Precision Products and are the focus of the rest of this paper. The overall HEFT design is described in more detail elsewhere. ${ }^{12}$

\section{PRODUCTION OF HEFT OPTICS}

A major accomplishment of the HEFT program has been the successful development of thermally-formed glass optics and an assembly method that is based on over-constraining these segmented optic substrates. In this section, we describe the production process of HEFT optics.

\subsection{Glass microsheets}

HEFT optics begin with widely available microsheet glass that has good initial properties such as microroughness, figure, and thickness uniformity. These substrates, which were developed for the flat panel display industry, are very light weight. Currently we are using $0.3 \mathrm{~mm}$ thick Schott AF-45 and D-263 microsheets, but we have also shown the viability of using thinner substrates. ${ }^{13}$ These substrates have low surface roughness of $\sim 3.5 \AA^{9}$ and provide a good surface for multilayer coatings. In this way, high x-ray reflectivity can be achieved at energies up to and even over $100 \mathrm{keV}$.

\subsection{Glass slumping}

Our approach is to thermally form these micro-sheets using standard quartz mandrels and commercially available ovens. Control of the temperature gradients in the oven is crucial since the viscosity of the glass, and thus the slumping rate, depend exponentially on temperature. ${ }^{14}$ Thus, the ovens are adapted to properly buffer the heat load from the input elements to provide a more uniform oven temperature. We begin the process by placing a glass micro-sheet on the mandrel inside of the oven. As the oven is heated to the appropriate temperature, the glass begins to form under the influence of gravity (see Fig. 2). The glass slowly assumes the large-scale 


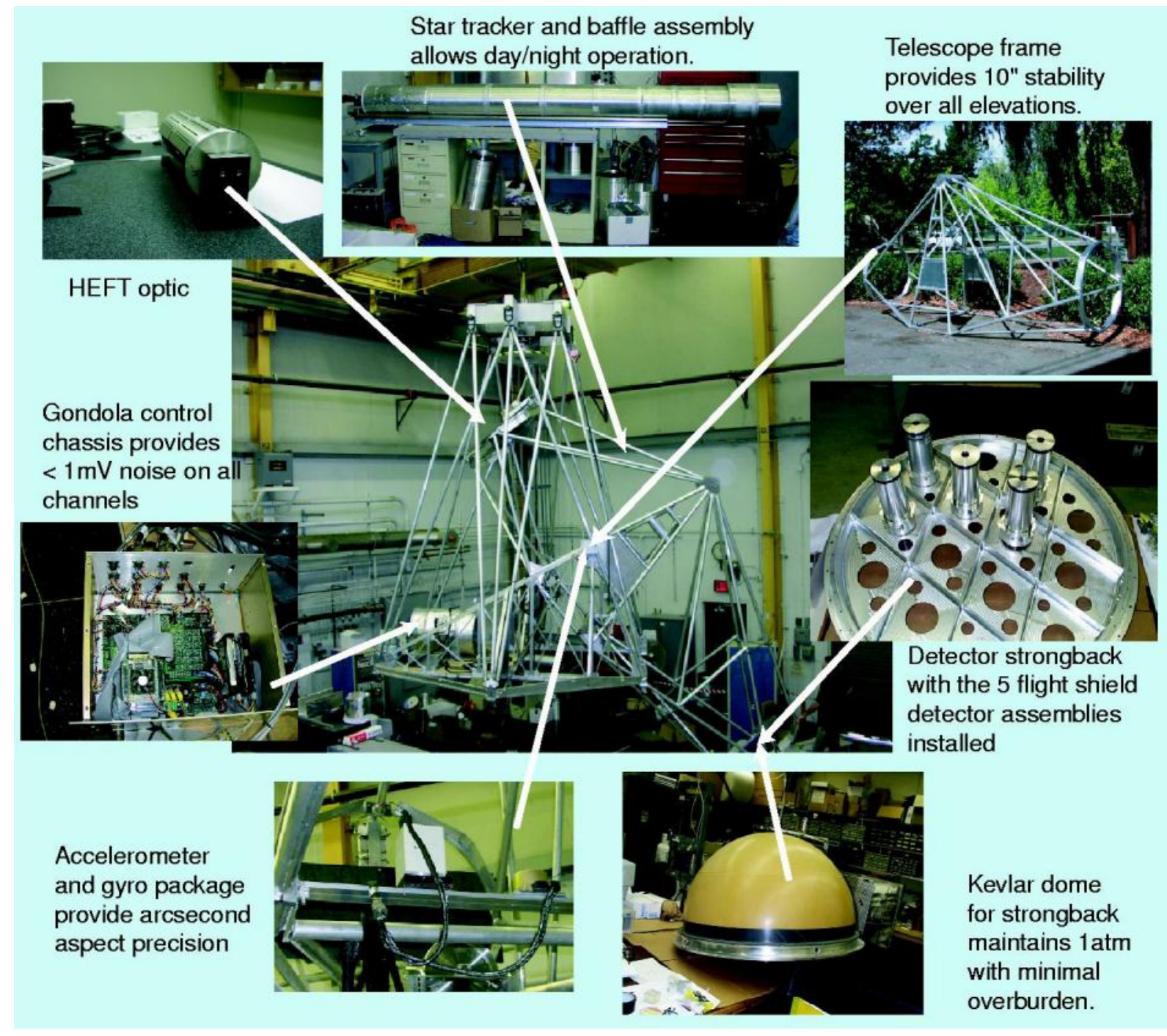

Figure 1. The major components of the HEFT experiment.

figure of the mandrel. Just before the glass touches the mandrel surface, the forming process is terminated by lowering the oven temperature. In this way, near net shaped optic substrates are produced without perturbing the excellent initial X-ray properties of the glass micro-sheet, even without the aid of highly polished and very expensive mandrels. We have studied the thermal slumping process in detail. This has culminated in the development of a complete analytic model based on viscous deformation theory to aid in optimizing the glass slumping process. ${ }^{14}$ This is essential in order to rapidly optimize oven parameters to obtain the optimal figure for a given glass radius.

\subsection{Glass Cutting}

Our ability to cut glass well is crucial to HEFT. The initial large sheets of glass must be cut to the appropriate size for slumping. After the glass pieces are slumped, they must again be cut along the optic axis as well as the curved azimuth direction to the appropriate size. This is necessary because the edges of the glass tend to warp during the forming process causing the overall performance of the shell to be degraded. Thus, we must isolate the higher performance central section of the slumped shell. We have been cutting the glass using a scribe and break technique. This technique is quick and easy and works very well for cutting the original flat 


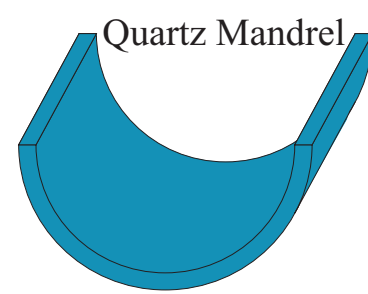

a)

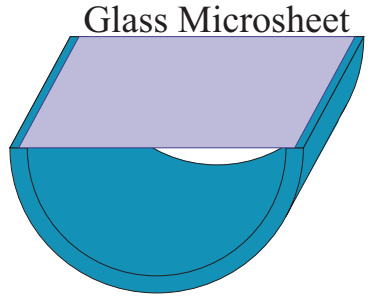

b)

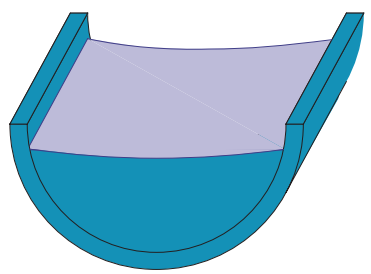

c)

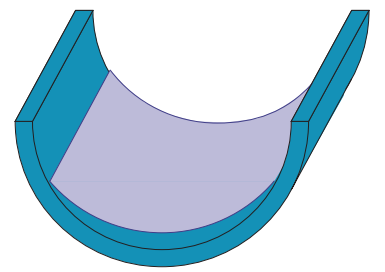

d)

Figure 2. a) The thermal forming process begins with a Quartz mandrel in a commercially available oven. b) An appropriately cut glass microsheet is placed on the mandrel. c) As the oven is heated to the appropriate forming temperature, the glass begins to form under the influence of gravity. d) Just before the glass touches the mandrel surface, the forming process is ended by decreasing the oven temperature, resulting in a high performance, near net shaped optic substrate.

glass microsheets to the correct size for slumping. The breaking of the slumped segments is aided by a hot wire that thermally shocks the piece causing it to break along the scribed cut. This also gives acceptable, but not perfect, edges for the slumped glass. The slumped glass is also much more difficult to cut than the flat glass, and small microcracks may be introduced along the cut edge form this process.

In order to achieve better quality control, we are moving toward cutting the slumped glass with a hot wire method used by W. Zhang at GSFC. ${ }^{15}$ In this method, a wire is heated from an applied current to a temperature below the melting temperature of the glass and brought in close proximity with the glass near the edge where the cut is to be initiated. The local heating of the glass and the fairly poor thermal conductance of the glass results in the high local stress that results in small fracture. The wire is then moved across the glass to propagate this fracture. With this process, no micofractures are introduced perpendicular to main fracture.

\subsection{Laser metrology of freestanding substrates}

An optical laser scanning apparatus, designed and built at Columbia's Nevis Laboratory and described more completely elsewhere ${ }^{16}$ is used to characterize free standing cylindrical substrates. The laser can be arbitrarily moved over the cylindrical surface, and deviations in the reflected beam are measured by a two-dimensional position sensitive diode (PSD) to an accuracy of approximately 5". This apparatus can accommodate both coated and uncoated cylindrical optic pieces and the full range of sizes used for HEFT. In the case of uncoated glass substrates, the reflections from the front and backside of the glass are almost equal. Thus, the backside of the substrate is painted with a mixture of matte black watercolor and sugar. This provides for a low-cost and easily removable anti-reflection coating that reduces reflections from the back surface to approximately $4 \%$ of the combined reflection intensity. From axial scan measurements at multiple azimuthal positions, the cylindrical surface can be fully reconstructed using software that removes errors in the shell alignment.

Being able to accurately assess the performance of the glass substrates immediately after they are formed and while they are still in their free state before mounting is crucial to quality control. Previously, no attempt was made to select high performance substrates and virtually $100 \%$ of the slumped glass was used. However, we are now beginning to become more selective in the pieces that we consider acceptable. The slumping recipes have continued to be more finely tuned to achieve better performing substrates. This fine tuning of the slumping parameters results directly from better understanding the correlation between the mounted and freestanding glass segments obtained through our prototype optics development program. Analysis of this laser metrology data can be used to predict the mounted performance of the substrate. In this way, we are able to continuously fine tune the oven slumping parameters in order to maintain the highest performance slumped glass.

\subsection{Multilayer coating}

Multilayer coatings are required to enhance the reflectivity with a broad energy acceptance. A multilayer structure is a stack of thin layers (typically several hundred layers) of alternating materials designed so that 
the small reflections from each layer add in phase. Depth-graded multilayers vary the bi-layer thickness so that different layers are optimized to reflect different wavelengths, providing broadband response. For W/Si multilayers, the absorption edge of $\mathrm{W}$ limits the effective operating energy range below $70 \mathrm{keV} .{ }^{9}$ To extend the bandpass to $100 \mathrm{keV}$ requires the use of Ni-based coatings on the inner shells that have the low graze angles necessary to reflect these high energy X-rays. ${ }^{17}$ The first two HEFT optics modules will use only W/Si coatings, but subsequent optics modules will take advantage of the NiV/Si coatings to extend the energy range up to 100 $\mathrm{keV}$. The substrates are coated at the Danish Space Research Institute (DSRI) at a rate almost twice that at which we can produce substrates. A more detailed description of HEFT multilayer coating is given elsewhere. ${ }^{9}$

\subsection{LVDT metrology of mounted substrates}

An air-bearing Linear Variable Differential Transformer (LVDT)* built by CPPI is used to perform low force surface metrology on the back surface of the mounted glass segments. The glass segments are very uniform in thickness as has been verified with our laser scanning apparatus, and thus, surface metrology performed on the back side of the glass is indicative of the front surface which serves to focus the X-rays. The LVDT is constant and near zero force metrology tool in that the diamond tipped probe contacts the back side of the optics using only about 1 gram of force, much less than would be necessary to deflect the optic surface. In the mode in which it is normally operated, the LVDT measurements are linear over a $100 \mu \mathrm{m}$ range with an accuracy of to $0.5 \%$ and a resolution of $15 \mathrm{~nm}$. LVDT data may be recorded for multiple axial scans at different azimuthal positions to generate a complete surface. The surface derivatives are clipped to remove any discontinuities in the surface profile introduced by particulates on the substrate, and a low pass filter is applied to the data to remove noise introduced in the data acquisition process.

The LVDT provides a very accurate assessment of the optic performance and has many advantages over other conventional contact metrology methods. The analysis of the LVDT surface measurements agrees quite well with both $8 \mathrm{keV}$ pencil beam X-ray and full illumination UV measurements performed on a three layer prototype optic module. These tests are described later in Section 4. With this tool, we are able to provide immediate verification of the optic performance. However, about 45 minutes is required to perform full metrology on each glass shell. While only partial metrology is necessary to accurately assess the shell performance, it is still time prohibitive to perform metrology on all 20 segments for each layer. Thus, only occasional partial metrology will be performed on selected segments for quality assurance.

\subsection{Optics assembly}

Larger scale figure errors including axial bows and small twists in the glass introduced during the thermal forming process limit the performance of the slumped segments in their free standing state. The glass substrates are also formed to a nearly cylindrical shape, while the telescope geometry requires that they be slightly conical in shape. Further, in order to increase the slumping production efficiency and minimize capitalization costs, we use only 24 mandrel sizes to produce the 144 different shell radii required by HEFT. However, with our mounting technique, the glass segments are constrained to precisely machined graphite spacers that run along the optical axis. In this way, the nominally cylindrical glass segments are forced to be conical, and in the process, the small twists and radial mismatches in the glass are removed. At the same time, the good X-ray properties of the glass such as smaller scale figure and micro-roughness are retained.

The assembly process begins with a precision mandrel. Graphite spacers are epoxied onto the mandrel and machined to the correct radius and angle as shown in Figure 3a. Mirror segments are then epoxied to the spacers to form the complete upper and lower layers (see Fig. 3b). In this process, very strong, thin, and uniform epoxy bondlines are achieved. After epoxy cure, the next layer of spacers is epoxied to the previous layer of glass. These spacers are machined with respect to the optic axis and another layer of glass is epoxied to the spacers (see Fig. 3c and d). In a similar way, subsequent layers are added to the optic assembly. The monolithic structures produced by this method provide the extreme mechanical robustness that is necessary for the instrument to survive multiple balloon campaigns.

${ }^{*}$ http://www.coloradoprecision.com/LVDT2 .htm 

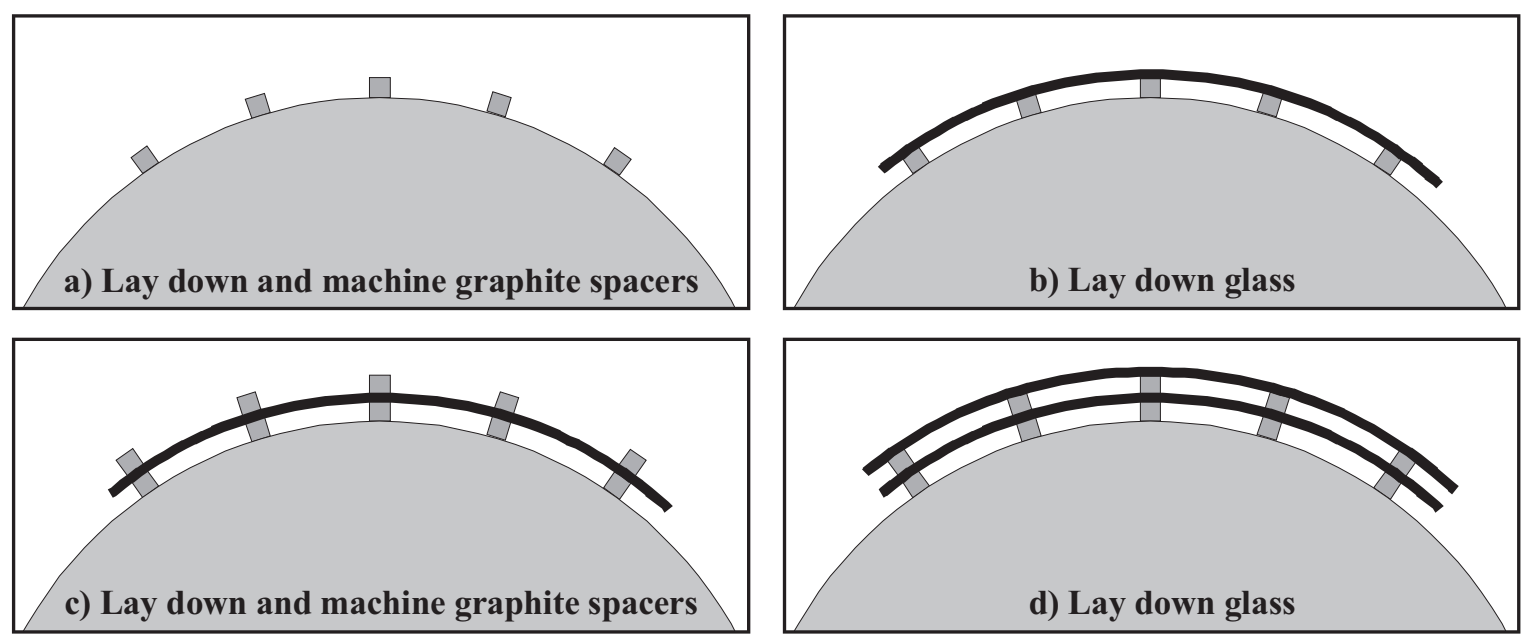

Figure 3. a) The assembly process begins by laying graphite spacers on a precision mandrel and machining them to the correct radius. b) Mirror segments are epoxied to the spacers. c) A new layer of spacers is machined to the first layer of glass and machined to the correct radius. d) The next layer of mirror segments are epoxied to the spacers.

In this assembly process, each layer of graphite spacers is precisely machined with respect to the optical axis, and thus, assembly errors do not stack up. The assembly apparatus has been designed and is continually being improved upon using a deterministic manufacturing philosophy that places great importance on understanding the cause and effect of assembly errors. In order to achieve the desired machine performance, attention must be given to process control at the sub-micron level. However, the assembly errors are quite small compared to the overall performance of our current substrates. The error in the machined spacers, along with other errors from calibration, measurements and fixturing, is estimated to contribute a total error of about 8 " to the entire assembly process. The actual performance of the optic is currently limited by the original figure of the free standing glass substrates. In the case of HEFT flight optics, the average overall optic performance will be about $60 "$ " However, much better thermally formed glass substrates with performance as low as $\sim 30$ " have been assembled in prototype development work. ${ }^{13}$

\section{HEFT PROTOTYPE DEVELOPMENT}

A full three layer, two-bounce test optic of full revolution was built to evaluate the assembly process and compare metrology techniques. Five quintant segments, each $20 \mathrm{~cm}$ in length, were used to form each upper and lower layer. Each of these $300 \mu \mathrm{m}$ thick conic segments has a nominal diameter of about $16 \mathrm{~cm}$ and subtend an angle of about 70 degrees (i.e., an arclength of about $10 \mathrm{~cm}$ ). Five graphite spacers, $16 \mathrm{~cm}$ in length each, were used to over-constrain each shell. Since no hard X-ray metrology was planned for this optic, only two of the fifteen sets of substrates were coated with multilayers. The two coated sets of mirrors demonstrate that the figure of the mounted optic shell and the epoxy bond strength is essentially unaffected by the coating. The performance of this optic was fully characterized using four independent metrology methods, all of which yielded consistent results.

In the first method, LVDT surface metrology (previously described in Section 3.6) was performed on the back side of each shell as the optic was being assembled. Since the glass is extremely uniform in thickness, the back surface metrology of the optic will reliably predict the performance of the front optical surface that is of interest. From the surface maps generated from this data, the focal plane image was simulated using a ray-trace code and is shown in Figure 4a. This surface topology data yields the most detailed information on the individual glass segments and is very useful in understanding the over-constraint mounting process.

In the second method, an ultra-violet (UV) full illumination test of a prototype optic was performed at 

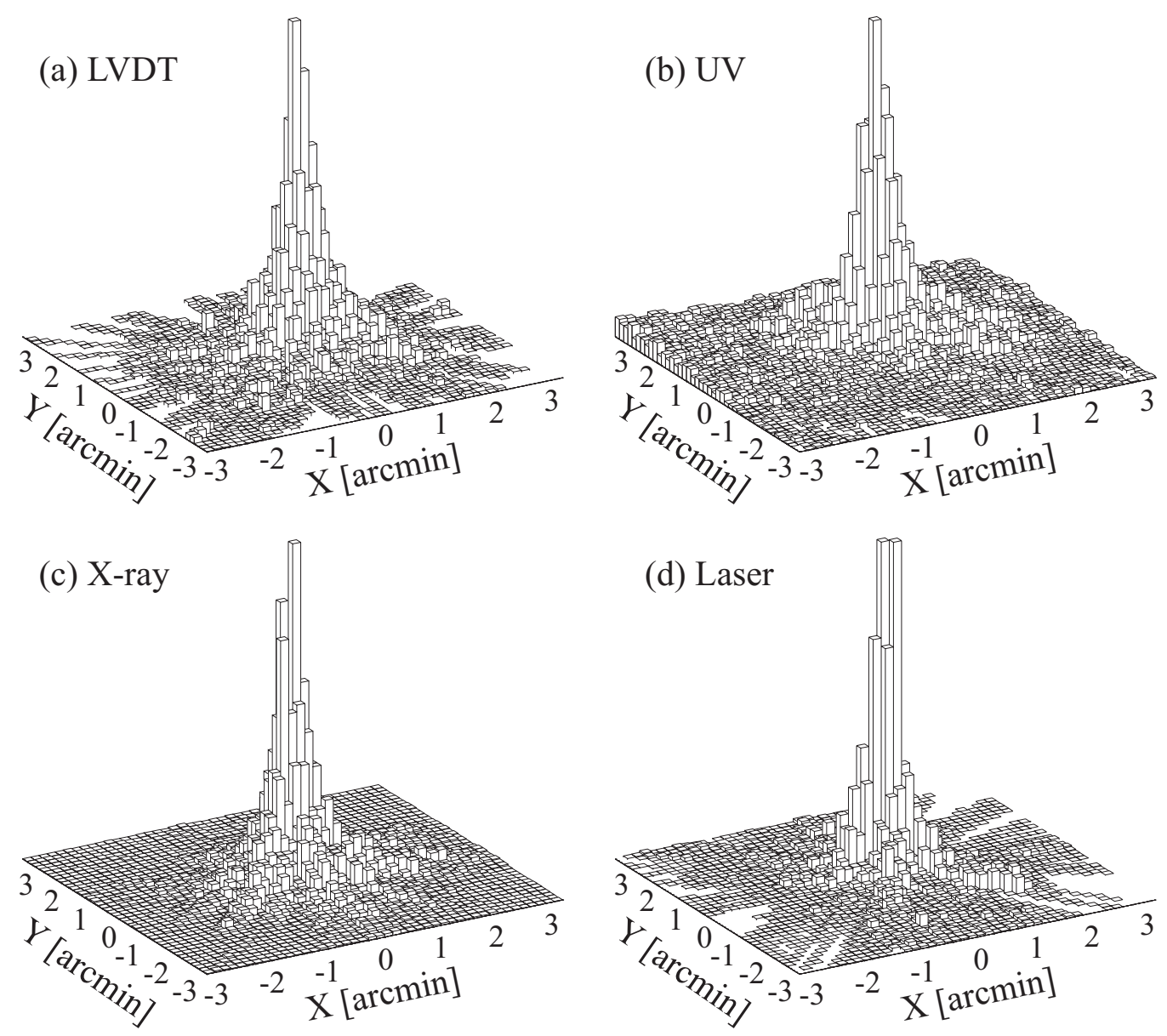

Figure 4. LVDT, UV, and X-ray metrology comparison for a complete two-bounce, three layer optic of full revolution. a) Point source image generated using a ray-trace code with LVDT surface metrology data.

b) Full illumination UV image (raw data with no background subtraction).

c) Composite image obtained from co-adding pencil beam scans performed using $8 \mathrm{keV}$ X-rays.

d) Point source image simulated using Laser surface metrology data of the premounted substrates.

the University of Colorado's Center for Astrophysics and Space Astronomy (CASA) ${ }^{\dagger}$ in Boulder, CO. The measurement was performed in a 'long-beam' vacuum tank that is shown in a drawing and photograph in Figure 5 (note that the drawing is not to scale). The UV source originates through a $100 \mu$ m diameter pin-hole on the side of the tank $2 \mathrm{~m}$ from one end of the tank. The source UV radiation is then reflected onto a parabolic mirror at one end of the tank by a collimating mirror centered on the optical axis of the tank. The parabolic mirror floods the vacuum tank with UV radiation directed parallel along optical axis of the tank. From the known quality of the mirrors and the size of the pin-hole, the quality of the parallel beam is estimated to be better than 10". The size of the source pin-hole was chosen in order to optimize the source intensity and angular size. The $6 \mathrm{~m}$ focal length of the test optic is longer than the usable length of the vacuum tank. Thus, the optic was positioned $3 \mathrm{~m}$ from the end opposite the parabolic mirror, and a gold folding mirror was used to reflect the UV radiation focused by the optic back onto a micro-channel plate (MCP) detector positioned next to the optic, as is indicated in Figure 5. The MCP detector, which was built by Siegmund Scientific ${ }^{\ddagger}$, has a sensitive area of $\pi \times(9 \mathrm{~mm})^{2}$ with $85 \mu \mathrm{m}$ resolution and operates with a quantum efficiency of about $5 \%$. A sheet of

\footnotetext{
${ }^{\dagger}$ CASA: http: /origins.colorado.edu

${ }^{\ddagger}$ Siegmund Scientific: http://siegmundsci.com/
} 


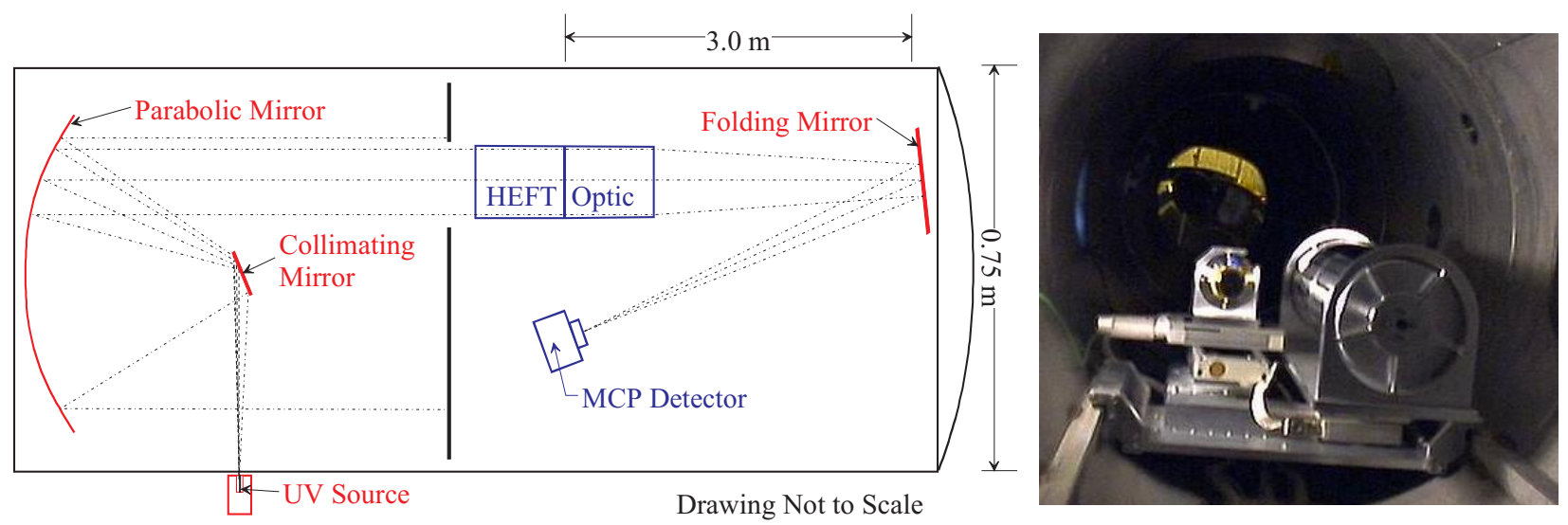

Figure 5. A drawing of the full illumination UV test facility (not to scale) is shown to the left. The prototype optic along with the MCP detector is shown in the photograph to the right. The parabolic mirror which floods the tank with parallel UV radiation is visible in the background.

Teflon with an opening for the optic was positioned in front of the optic and MCP to shield the MCP from background UV radiation. The source collimating mirror and parabolic reflection mirror were both precisely aligned by the CASA staff. The optic was initially aligned along the optical axis of the UV chamber with the aid of a white light source by manually adjusting a tilt stage to align the inclination of the optic and by remotely adjusting a Klinger rotation stage to align the rotation angle of the optic. In this way, the optic was estimated to be aligned with the optical axis to better than an arcminute. From raytrace calculations it is found that about $10 \%$ of the photons will be lost for every arcminute the photons are incident off the optical axis of the telescope. This loss is due to obscuration by the mandrel and the other shells. The shape of the image will also become slightly asymmetric for off-axis incidence angles, but the overall HPD performance will largely remain unaffected for off-axis incidence angles up to several arcminutes. Thus, alignment of the optic to better than a few arcminutes is not necessary to obtain an accurate determination of the optic performance. The folding mirror was aligned manually with the aid of a laser originating from the pin-hole source such that it focused the source onto the center of the MCP detector. Using a Ne UV source, the depth of focus was adjusted by moving the MCP detector on a Klinger translation stage along the optical axis as reflected by the folding mirror. A high vacuum was maintained using a liquid Helium cryogenic pump, and hollow cathode Argon, Neon and Helium sources were used during these measurements.

The resulting image obtained directly from this measurement is shown in Figure 4b. While this method provides no detailed information on the individual optic components, it unambiguously provides a 'what you see is what you get' result for the image requiring essentially no intermediate data analysis steps. However, while characteristics such as the image full width at half maximum (FWHM) are quite easily and accurately determined, a certain level of uncertainty exists in predicting the image performance in the form of the HPD due to the background level involved, about one percent of the image peak intensity. The uncertainty in extracting the HPD could be decreased with a precise calibration of the source intensity, but this is very challenging and was avoided because it would have added a significantly more complex element to this measurement. The UV metrology setup also has the advantage that, once everything is in place, multiple measurements can be relatively quickly performed to gain a more complete understanding of the optic performance. In this way, it was possible to directly evaluate the off-axis response of this prototype optic and compare it to ray-trace simulations. This is opposed to simply relying on raytrace simulations as is often done because of the prohibitively long time required for other metrology methods such as X-ray pencil beam scans. Further, depth of focus studies can be quickly performed to optimize the focal distance of the telescope. Both off axis and depth of focus measurements of the telescope confirm that the optic is indeed performing as expected.

In the third method, $8 \mathrm{keV}$ X-ray pencil beam scans were performed at $2.5^{\circ}$ increments over each of the three layers. The test setup is shown in the picture in Figure 6a, and the resulting composite image is shown 

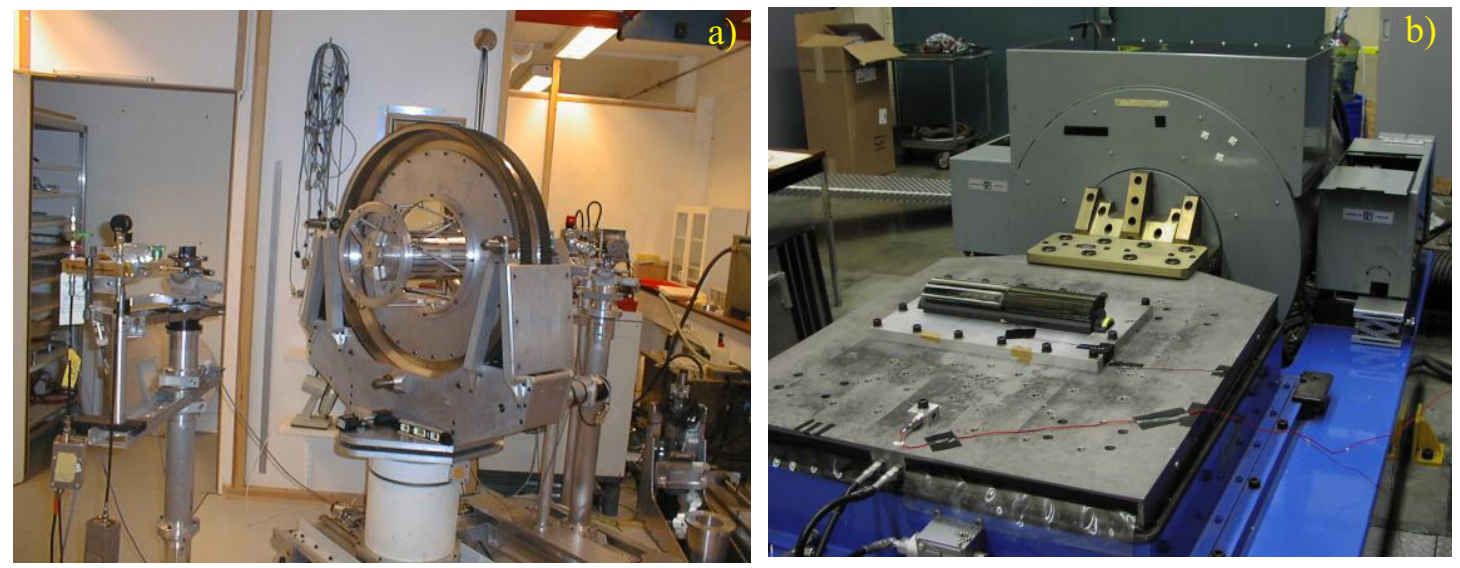

Figure 6. a) Metrology on a HEFT prototype where $8 \mathrm{keV}$ X-rays pencil beam scans were performed at $2.5^{\circ}$ increments over each of the three layers. b) Shake test performed on a diferent prototype to demonstrate the structural integrity of our mounting approach.

in Figure 4c. This metrology method yields more information than the UV full illumination method by giving the composite two-bounce performance of the upper and lower shells at multiple azimuthal positions. In this way, general comparisons such as the performance near and further away from the spacers can be evaluated. In determining the performance of an optic, the $8 \mathrm{keV}$ X-rays have been shown to be a reliable substitute for the 20-70 keV design energy of the telescope because the performance is dominated by figure error and not by energy dependant scattering. ${ }^{18}$ This metrology has other advantages in that the background is extremely low and there are only small systematic uncertainties $(\sim 5 ")$ associated with co-adding the individual scans due to misalignment of the optic during the measurements. Thus, the X-ray pencil beam scans provide the most accurate determination of the image HPD.

Finally, in the fourth method, the performance of the optic was predicted using the surface maps of the unmounted, freestanding substrates obtained using the laser scanning apparatus (previously described in Section 3.4). In the analysis of this data, it is assumed that the mounting process perfectly constrains the nominally cylindrical substrates to the appropriate conic shape, and in the process removes any twist or radius mismatch in the freestanding substrate. In this way, the relative error in the overall slope and radial offset of each laser measurement along the shell optic axis is removed to simulate the mounting process. The 30 segments that make up this test telescope are then virtually mounted in their appropriate positions and raytrace calculations are performed. The image from a raytrace of this data is shown in Figure 4d. This simple analysis procedure of the freestanding substrate metrology data provides a remarkably accurate prediction of the overall optic performance.

The good agreement between the X-ray and UV measurements indicate that no difficulties exist in properly aligning the optic for the X-ray pencil beam scans. The analysis of the LVDT surface measurements can also be relied upon to give accurate predictions of the image performance since it agrees quite well with both the X-ray and UV measurements. Each of these three methods have different strengths, and together, they provide for a very clear and self-consistent understanding of the performance of an assembled optic. Further, the excellent agreement between the simulated performance obtained from the laser data and the actual measured performance obtained from the LVDT, UV and X-ray data demonstrates that the laser data can be used as a powerful tool to predict the performance that an ensemble of individual segments will provide when mounted using our assembly method. This test optic clearly demonstrates our ability to build a two bounce optic of full revolution using a relatively large number of segments that all consistently and accurately focus onto a single focal plane.

We have also tested past prototype optics for structural integrity. One optic module was tested in all three axes to the Delta IV qualification level vibration spectrum with no apparent degradation to the glass. This test 


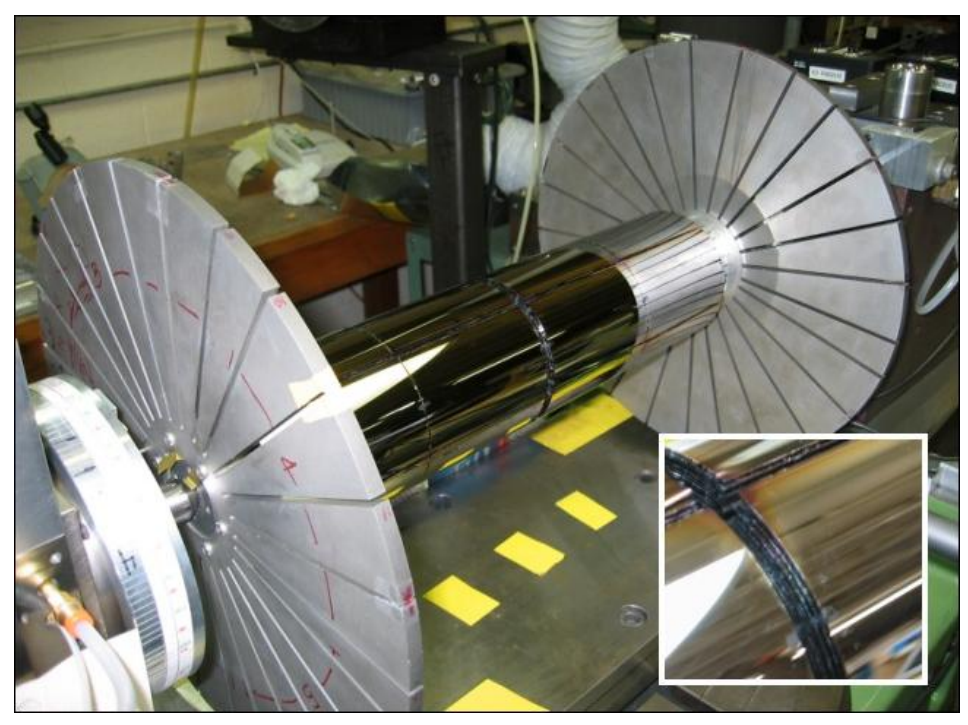

Figure 7. Photograph of the first HEFT flight optic being assembled at CPPI.

setup is shown in Figure 6b. The lowest natural frequency of the glass between supports is approximately 700 $\mathrm{Hz}$. This high natural frequency will not couple with the low frequency spectrum typically found on rockets and even lower frequencies associated with balloon missions. The greatest potential for damage is a high shock load, greater than $25 \mathrm{~g}$ 's. This gives a reasonable margin of safety over the shock load expected from the parachute of $\sim 10$ gs. Further, the mounting system for the entire optic will be designed to attenuate much of the introduced shock load using passive damping and compliant structural supports where appropriate. This gives us confidence that the flight optics we are building will able to survive multiple balloon campaigns.

In further prototype development, we have shown that by cutting substrates down to $10 \mathrm{~cm}$ in length we can achieve arcminute performance. Specifically, a total of 34 individual $10 \mathrm{~cm}$ long segments were mounted in a two-bounce configuration with an average performance of $54 \pm 5$ " obtained from X-ray measurements. ${ }^{13}$ This length and type of substrate has been adopted for the first HEFT optics modules.

\section{FLIGHT MODULE ASSEMBLY PROGRESS}

We are well underway constructing the first HEFT flight optic as can be seen in the photograph of the optic in Figure 7. Sample LVDT metrology from this first telescope is shown in Figure 8 along with preliminary performance analysis. Figures $8 \mathrm{a}-\mathrm{d}$ show the three-dimensional surfaces for four segments in one quintant section. The axial LVDT scans were performed every $7.5^{\circ}$, and a fifth-order Butterworth filter with a frequency cutoff of 1 cycle per $\mathrm{cm}$ has been applied to the raw data to remove noise introduced during data acquisition. The $10 \mathrm{~cm}$ long a and b segments form the upper layer and the $10 \mathrm{~cm}$ long $\mathrm{c}$ and $\mathrm{d}$ segments form the lower layer of the two-bounce optic. A raytrace program was used to simulate the image generated by these four segments that is shown in Figure 8e. The fraction of enclosed energy from this image is plotted versus performance for this entire prototype optic in Figure $8 \mathrm{f}$. In order to focus more on the axial figure of the segments, the LVDT axial scans are also plotted in the two-dimensional plot in Figure 8e.

\section{CONCLUSIONS}

In our assembly process, each optic layer is precisely assembled with respect to the spindle axis, and thus, assembly errors do not stack up. Immediate verification of performance through mechanical metrology allows for continual quality assurance throughout the assembly process. The assembly procedure is not highly complicated and can be easily performed by low tech personnel. The performance of our assembly method (8") itself easily exceeds the requirements for HEFT. Currently, the total performance is completely limited by the figure of the 

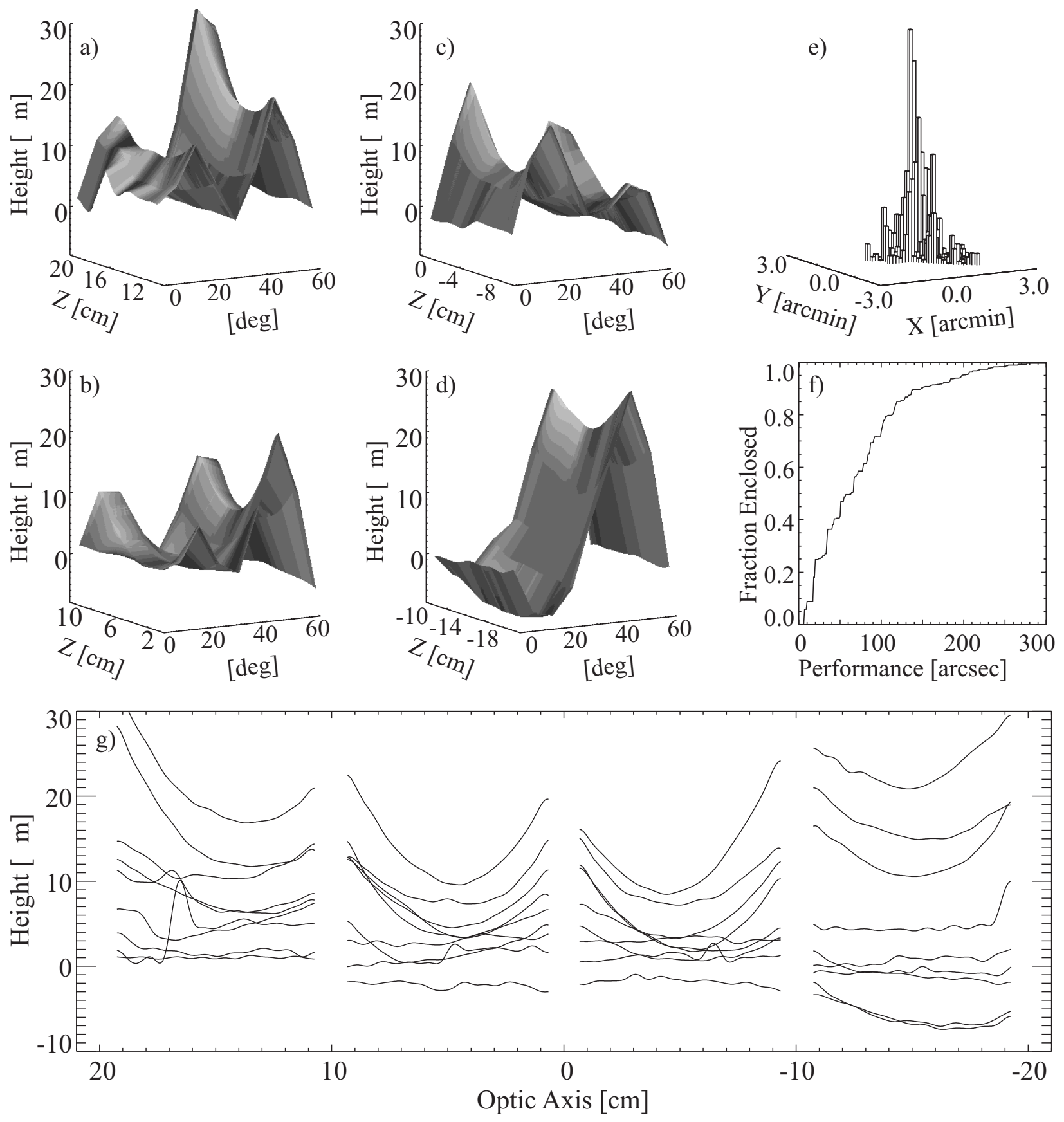

Figure 8. The LVDT data for one quintant section is shown as an example of the performance of the first HEFT flight module that is under construction at CPPI. The upper segments are plotted in a) and b), and the lower segments are plotted in c) and d). The resulting image generated using this data and a raytrace program is shown in e). From this, the fraction of enclosed energy is plotted versus performance in $\mathrm{f}$ ). The LVDT axial scans are also shown in the two-dimensional plot in g) for this set of four segments. 
thermally formed glass. HEFT will directly benefit from our independent research program to produce even higher performance substrates. ${ }^{13}$ In this way, we can expect to achieve improved performance for subsequent HEFT modules.

\section{ACKNOWLEDGMENTS}

This technical progress described in this paper could not have been made without the expert and tireless work of our technical staff. In particular, Marcela Stern was essential in defining the slumping techniques. We are very grateful to CASA at the University of Colorado for hosting us as guests at their excellent long-beam UV facility. This work is supported by a NASA grant to Columbia University: NAG5-5260 (Thermally Formed Optics for X-Ray and Gamma-Ray Astronomy), and by NASA grants to the California Institute of Technology with subawards to Columbia: Caltech No. 1019776 (High Energy Focussing Telescope) and Caltech No. 1046806 (Critical Technologies for theConstellation Hard X-Ray Telescope).

\section{REFERENCES}

1. P. Serlemitsos et al., "The x-ray telescope on board ASCA," PASJ 47(1), pp. 105-114, 1995.

2. P. Serlemitsos, "Foil X-Ray Mirrors for Astro-E," American Astronomical Society Meeting 30, pp. 1373-+, Dec. 1998.

3. N. Westergaard, J. Polny, F. Christensen, H. Noergaard-Nielsen, and H. Schnooper, "Optical measurements of the xspect mirrors and the assembled mirror modules for the sodart $\mathrm{x}$-ray telecope on the spectrum-xgamma satellite," Proc. SPIE 3113, p. 458, 1997.

4. J. Polny, N. Westergaard, F. Christensen, H. Noergaard-Nielsen, and H. Schnooper, "Production, assembly, and alignment of the xspect mirror modules for the sodart x-ray telescope on the spectrum roetgen gamma satellite," Proc. SPIE 3113, p. 349, 1997.

5. B. Aschenbach et al., "Imaging performance of the XMM-Newton x-ray telescopes," Proc. SPIE 4012, pp. 731-739, 2000.

6. M. Weisskopf et al., "Chandra X-ray Observatory (CXO): overview," Proc. SPIE 4012, pp. 2-16, 2000.

7. C. Hailey, S. Abdali, F. Christensen, W. Craig, T. Decker, F. Harrison, and M. Jimenez-Garate, "Substrates and mounting techniques for the high-energy focusing telescope (heft)," Proc. SPIE 3114, p. 535, 1997.

8. F. A. Harrison, A. E. Bolotnikov, H. C. Chen, W. R. C. III, and S. M. Schindler, "Characterization of a high spectral resolution cadmium zinc telluride pixel detector for astrophysical application," Proc. SPIE 4851(101), 2002.

9. C. P. Jensen, H. Chen, F. E. C. K. Madsen, and E. Ziegler, "Coating of the heft telescope mirrors: method and results," Proc. SPIE 4851(79), 2002.

10. P. Mao, F. Harrison, Y. Platonov, D. Broadway, B. Degroot, F. Christensen, W. Craig, and C. Hailey, "Development of grazing incidence multilayer mirrors for hard x-ray focusing telescopes," Proc. SPIE 3114, p. 526, 1997.

11. P. Mao, F. Harrison, D. Windt, and F. Christensen Applied Optics 38, p. 22, 2000.

12. F. Harrison, S. Boggs, A. Bolotnikov, F. Christensen, W. Cook, W. Craig, C. Hailey, M. Jimenez-Garate, P. Mao, S. Schindler, and D. Windt, "Development of the high-energy focusing telescope (heft) balloon experiment," Proc. SPIE 4012, p. 693, 2000.

13. J. Koglin, H. Chen, F. Christensen, J. Chonko, W. Craig, T. Decker, K. Gunderson, C. Hailey, F. Harrison, C. Jensen, M. Sileo, D. Windt, and H. Yu, "Development of precision hard x-ray multilayer optics with sub-arcminute performance," Proc. SPIE 4851(74), 2002.

14. M. Jimenez-Garate. PhD thesis, Columbia PhD. (physics), 2001.

15. W. Zhang private communication.

16. M. Jimenez-Garate, W. Craig, C. Hailey, F. Christensen, and A. Hussain Opt. Eng. 39(11), p. $2982,2000$.

17. D. L. Windt, J. Koglin, C. J. Hailey, E. Ziegler, F. E. Christensen, and F. A. Harrison, "Hard x-ray multilayer structures optimized for use above $100 \mathrm{kev,"} \mathrm{Proc.} \mathrm{SPIE} \mathrm{4851(70),} 2002$.

18. W. Craig, C. Hailey, M. Jimenez-Garate, D. Windt, F. Harrison, P. Mao, F. Christensen, and A. Hussain Optics Express 7, p. 178, 2000. 